\title{
Heterodyne lock-in thermal coupling measurements in integrated circuits: Applications to test and characterization
}

\author{
J. Altet, ${ }^{1}$ E. Aldrete-Vidrio, ${ }^{1}$ D. Mateo,${ }^{1}$ A. Salhi, ${ }^{2}$ S. Grauby, ${ }^{2}$ W. Claeys,${ }^{2}$ S. Dilhaire, ${ }^{2}$ \\ $X$. Perpiñà, ${ }^{3}$ and $X$. Jordà ${ }^{3}$ \\ ${ }^{1}$ Department of Electronic Engineering, Universitat Politècnica de Catalunya, C/. Jordi Girona 1-3, \\ Campus Nord UPC, 08034 Barcelona, Spain \\ ${ }^{2}$ CPMOH, Université Bordeaux I, 351 Cours de la Libération, F-33405 Talence Cedex, France \\ ${ }^{3}$ Centre Nacional de Microelectrónica, Campus UAB, Bellaterra, 08193 Barcelona, Spain
}

(Received 29 October 2008; accepted 4 January 2009; published online 2 February 2009)

\begin{abstract}
Heterodyne strategies can be used to characterize thermal coupling in integrated circuits when the electrical bandwidth of the dissipating circuit is beyond the bandwidth of the thermal coupling mechanism. From the characterization of the thermal coupling, two possible applications are described: extraction of characteristics of the dissipating circuit (the determination of the center frequency of a low-noise amplifier) and the extraction of the thermal coupling transfer function. (C) 2009 American Institute of Physics. [DOI: 10.1063/1.3073963]
\end{abstract}

Thermal coupling characterization in an integrated circuit (IC) involves three different entities: devices that dissipate a considerable amount of power, the IC structure that conducts this dissipated heat from the source to the sink, and finally, a temperature sensor. Practical applications consist of extracting the information from one of these entities when the other ones are already known. ${ }^{1-3}$

The use of lock-in amplifiers allows measuring the amplitude and phase of one spectral component of the sensed temperature $^{4,5}$ when the dissipating device operates in a modulated regime. Being very sensitive to small temperature increases and robust to noise, this sensing strategy is suitable for temperature measurements in low-power circuits. ${ }^{6}$

Measuring in the frequency domain, one of the most important issues is the maximum frequency at which temperature measurements can be made, being the bottleneck imposed by the particular physical behavior of the heat transfer within the IC structure. This transfer function, which relates the power dissipated by the circuit with the measured temperature, has a lowpass filtering response. The bandwidth of the temperature sensor depends on its electronic design (in this work we assume that this value does not limit the temperature measurements). Finally, the involvement of the dissipating devices can be classified into two classes. One scenario occurs when the transfer function of the thermal coupling mechanism falls into the electrical bandwidth of the dissipating devices [Fig. 1(a)]. Then, it is possible to generate modulated power dissipation, which falls into the thermal coupling mechanism bandwidth with just one sinusoidal electrical excitation. ${ }^{4}$

Another case is depicted in Fig. 1(b), where the electrical transfer function of the dissipating circuit/device does not overlap with the transfer function of the thermal coupling. In this case, if we drive the circuit with one sinusoidal voltage generator, only the dc component of the dissipated power will generate a measurable temperature increase.

Nevertheless, if this linear circuit is excited with two sinusoidal voltage functions of frequencies $f_{1}$ and $f_{2}$, its dissipated power has many spectral components, e.g., dc, $2 f_{1}$,
$2 f_{2}$, and the heterodyne components $f_{1}+f_{2}$ and $f_{1}-f_{2}\left(f_{1}\right.$ $\left.>f_{2}\right){ }^{7}$ The values of $f_{1}$ and $f_{2}$ may be beyond the cutoff frequency of the thermal coupling mechanism, but the component at $f_{1}-f_{2}$ generates a temperature increase measurable with a lock-in amplifier and can be used to characterize thermal coupling.

The following two sections show how this strategy can be used to characterize the thermal coupling in the frequency domain for the case depicted in Fig. 1(b). The first example shows how it is possible to obtain information about the dissipating circuit: the center frequency of a $1 \mathrm{GHz}$ lownoise amplifier (LNA) is obtained by measuring the spectral component of the temperature increase at $1 \mathrm{kHz}$. The second example explains how to obtain the transfer function of the thermal coupling mechanism of a thermal test die, behaving like typical vertical power devices.

When the dissipating circuit is a linear amplifier and it is driven by two sinusoidal voltage signals of amplitude $A$ and frequencies $f_{1}$ and $f_{2}$, the amplitude of the power dissipated by its devices at $\left(f_{1}-f_{2}\right)$ linearly depends on the gain of the amplifier at frequencies $f_{1}$ and $f_{2}$. If both frequencies are close enough, these two gains have almost the same value and the amplitude of the power dissipated at $\left(f_{1}-f_{2}\right)$ is proportional to the gain to either $f_{1}$ or $f_{2}{ }^{7}$

Therefore, if two tones of frequencies $f_{1}$ and $f_{2}$ are applied to a linear amplifier and the values of $f_{1}$ and $f_{2}$ are swept across its operational bandwidth, keeping the value of $\left(f_{1}-f_{2}\right)$ constant, e.g., $1 \mathrm{kHz}$, the amplitude of the spectral component of the sensed temperature at $1 \mathrm{kHz}$ is an observable of the frequency response since its amplitude is proportional to the gain of the amplifier at frequencies $f_{1}$ and $f_{2}$.

For the experimentation we have designed a singleended cascoded LNA with inductive source degeneration ${ }^{8}$ formed by two metal oxide semiconductor (MOS) transistors: active and cascoded. The chip photograph is shown in Fig. 2 (0.25 $\mu \mathrm{m}$ MS/rf deep $n$-well CMOS (complementary MOS) technology, with an area of $1.25 \times 1.25 \mathrm{~mm}^{2}$ ), and the location of transistors MNC (cascoded) and MNA (active) is highlighted (they are separated $350 \mu \mathrm{m}$ ). 

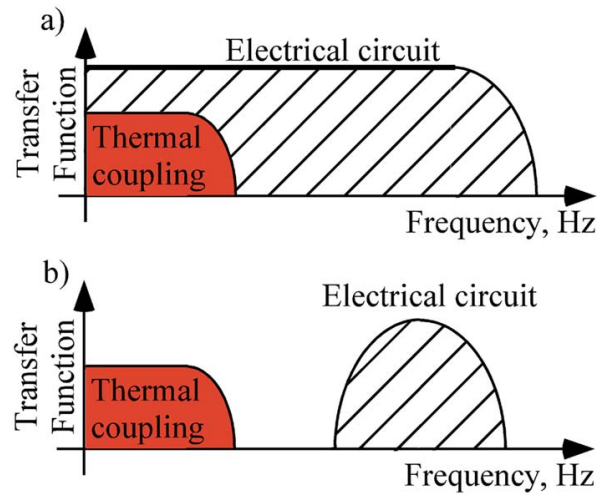

FIG. 1. (Color online) Transfer function of the thermal coupling: low pass filter behavior. (a) The dissipating device/circuit has low pass filter behavior. (b) The dissipating device/circuit has bandpass filter behavior and does not fall into the thermal coupling mechanism bandwidth.

We have performed temperature measurements with a laser reflectometer. ${ }^{9}$ In our particular setup, the area lighted by the laser has a radius of less than $5 \mu \mathrm{m}$, which is focused on the silicon surface at $25 \mu \mathrm{m}$ from transistor MCN (see Fig. 2). The signal provided to the photodiode is sent to the lock-in amplifier, which measures the amplitude and phase of the desired spectral component of the signal provided to the reflectometer, which is proportional to the amplitude and phase of the same spectral component of the temperature increase at the area illuminated by the laser. In this particular setup, this temperature increase is generated by the power dissipated in transistor MCN.

The LNA is driven with two tones of frequencies $f_{1}$ and $f_{2}$ (input power $=-3 \mathrm{dBm}$ ). The values of $f_{1}$ and $f_{2}$ have been swept in such a way that $f_{2}$ goes from $500 \mathrm{MHz}$ to 1.6 $\mathrm{GHz}$ and $\Delta f=f_{1}-f_{2}$ is equal to $1012 \mathrm{~Hz}$. With the lock-in amplifier, we have observed the amplitude of the spectral

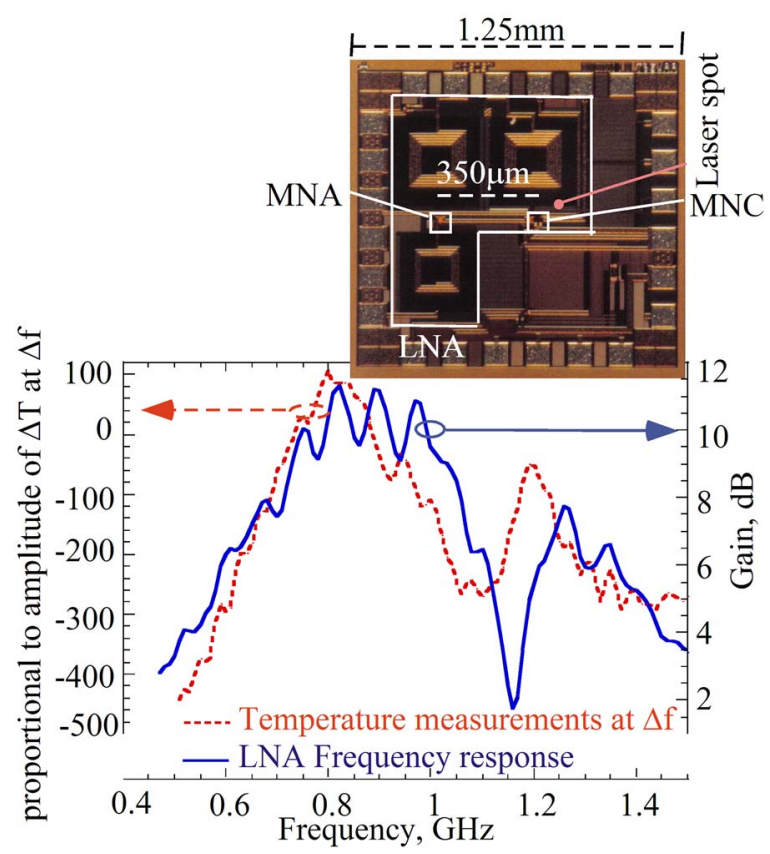

FIG. 2. (Color online) LNA chip die photograph. Frequency response (amplitude in $\mathrm{dB}$ ) of the amplifier. Amplitude of the temperature increase measured at the frequency $\Delta f=\left(f_{1}-f_{2}\right)$ as a function of the value of $f_{2}$.

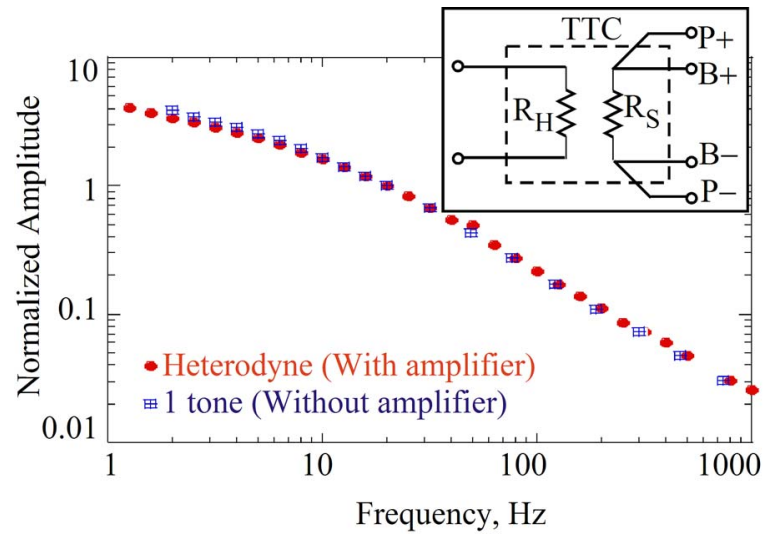

FIG. 3. (Color online) Experimental thermal coupling response between $R_{H}$ and $R_{S}$ and schematic equivalent circuit of the TTC (inset).

component of the temperature increase at this frequency $(1012 \mathrm{~Hz})$ as a function of the frequency $f_{2}$.

Figure 2 compares the frequency response of the amplifier (right $y$ axis) with the measured amplitude of the spectral component of the temperature increase at the frequency $\Delta f$ (left $y$ axis). For the latter case, the $x$ axis shows the value of $f_{2}$.

The signal obtained from temperature measurements has good agreement with the frequency response of the amplifier in the frequency range from $500 \mathrm{MHz}$ to $1.1 \mathrm{GHz}$. Electrical measurements provide a center frequency of $900 \mathrm{MHz}$, whereas temperature measurements indicate a center frequency of $830 \mathrm{MHz}$. Electrical measurements show a notch in the frequency response at $1.16 \mathrm{GHz}$, whereas the notch appears at $1.10 \mathrm{GHz}$ in the temperature measurements.

The inset of Fig. 3 shows the equivalent circuit schematic of a thermal test chip (TTC) devoted to thermal experiments. ${ }^{10}$ It contains a $61.4 \Omega$ polysilicon resistor and a $640 \Omega$ platinum resistor. The polysilicon resistor $\left(R_{H}\right)$ is used as a dissipative device and it is formed by the parallel connection of 130 polystrips homogeneously placed over the IC surface $\left(5.4 \times 5.4 \mathrm{~mm}^{2}\right)$. This arrangement shows the same thermal behavior than typical vertical power devices (insulated gate bipolar transistors, IGBT, vertical diffuse MOS transistors, VDMOS, and fast recovery diodes FRD). The platinum resistor $\left(R_{S}\right)$ is placed at the center of the silicon die with an area of $0.7 \times 0.7 \mathrm{~mm}^{2}$. Biased with a dc of $15 \mathrm{~mA}$ ( $B$ connectors), it is used as a resistive thermal device (RTD) temperature sensor ( $P$ connectors).

For validation purposes, we have obtained the thermal coupling transfer function between both resistors in two configurations: (i) $R_{H}$ is driven with a sinusoidal voltage generator of frequency $f$ and the temperature at $R_{S}$ is sensed at the frequency $2 f$ [such as Fig. 1(a)]; (ii) $R_{H}$ is driven with an audio amplifier whose band ranges from $15 \mathrm{~Hz}$ to $70 \mathrm{kHz}$ [Fig. 1(b) for frequencies lower than $15 \mathrm{~Hz}$ ]. To extract the thermal coupling transfer function between both resistors for frequencies lower than $15 \mathrm{~Hz}$, we have biased $R_{H}$ with two tones of frequencies $f_{1}$ and $f_{2}\left(f_{1}=f_{2}+\Delta f\right)$. With $f_{2}$ $=32 \mathrm{kHz}$ and $\Delta f$ ranging from $1 \mathrm{~Hz}$ to $1 \mathrm{kHz}$, the temperature at $R_{H}$ was measured at the frequency $\Delta f$. Figure 3 shows the normalized amplitude of the spectral component of the temperature sensed with the two configurations as a function of the frequency at which temperature is measured. As can be seen, there is a very good agreement between both plots, 
showing that with this heterodyne strategy, the thermal coupling can be characterized for the case depicted in Fig. 1(b).

This work has been partially supported by the Spanish Ministerio de Educación y Ciencia (under Contract No. TEC2008-01856 and GICSERV Program), FEDER funds, and the Consejo Superior de Investigaciones Científicas (CSIC) (under contract "Junta para la Ampliación de Estudios," JAE-Doc). The authors thank Mr. Marvin Onabajo for the rich discussion about the work.

\footnotetext{
${ }^{1}$ A. Altes, R. Tilgner, M. Reissner, G. Steckert, and G. Neumann, Microelectron. Reliab. 48, 1273 (2008).

${ }^{2}$ M. Rencz, A. Poppe, E. Kollar, S. Ress, and V. Szekely, IEEE Trans. Compon. Packag. Technol. 28, 51 (2005).
}

${ }^{3}$ C. W. Meyer, W. A. Kimes, and D. C. Ripple, Meas. Sci. Technol. 19, 055202 (2008)

${ }^{4}$ X. Perpiña, X. Jorda, M. Vellvehi, J. Altet, and N. Mestres, J. Phys. D 41, 155508 (2008).

${ }^{5}$ X. Perpiña, J. Altet, X. Jorda, M. Vellvehi, J. Millan, and N. Mestres, IEEE Electron Device Lett. 29, 1142 (2008).

${ }^{6}$ O. Breitenstein, M. Langenkamp, F. Altmann, D. Katzer, A. Lindner, and H. Eggers, Rev. Sci. Instrum. 71, 4155 (2000).

${ }^{7}$ D. Mateo, J. Altet, and E. Aldrete-Vidrio, Microelectron. J. 38, 151 (2007)

${ }^{8}$ T. H. Lee, The Design of CMOS Radio-Frequency Integrated Circuits (Cambridge University Press, Cambridge, 1998)

${ }^{9}$ J. Altet, W. Claeys, S. Dilhaire, and A. Rubio, Proc. IEEE 94, 1519 (2006)

${ }^{10}$ X. Perpiñà, Power Devices Electrothermal Characterisation by Optical Techniques: An Experimental Approach to Analyse Internal Electrothermal Phenomena at Device Level (VDM, Saarbrücken, 2008). 